\title{
Interview mit ASP-Mitglied Elisabeth Ansen Zeder
}

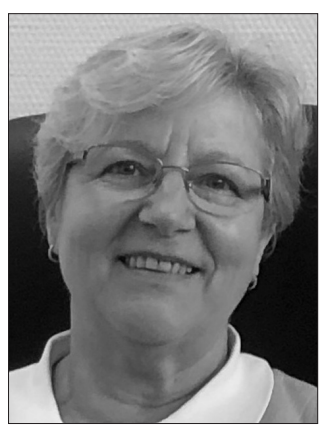

Elisabeth Ansen Zeder
Welche Motivation gab es für Sie, den Beruf der Psychotherapeutin auszuwählen? Das waren zutiefst menschliche Gründe. Ich bin in Strassburg aufgewachsen, in einem recht bodenständigen Umfeld, doch einige familiäre Probleme waren Antrieb für mich, die Psyche verstehen $\mathrm{zu}$ wollen. Meine Lebenserfahrung war also meine Hauptmotivation.

\section{Wie sieht Ihr beruflicher}

Hintergrund/Werdegang aus?

$\mathrm{Da}$ ich als Erzieherin für Kleinkinder angefangen habe, komme ich aus dem Bereich der direkten Betreuung. Ich habe als Facherzieherin in verschiedenen Einrichtungen, vornehmlich in der französischen Kinderhilfe gearbeitet. Ich habe auch einige Jahre im staatlichen Bildungswesen in Frankreich und Belgien gearbeitet, bevor ich 1999 in die Schweiz kam. Als ich in der Region Rhône-Alpes lebte, machte ich 1991 bei den Professoren René Roussillon und Jacques Hochmann in Lyon meinen DEA-Abschluss in Psychologie und klinischer Psychopathologie. Ich habe eine Psychoanalyse nach Lacan durchgeführt, mich in Transaktionsanalyse fortgebildet und machte später meinen Doktor in klinischer Psychologie bei der Professorin Evelyne Thommen. In diesem Rahmen erforschte ich den Resilienzprozess bei Geschwistern von Menschen mit geistiger Behinderung. Ich habe auch einen Kurs zur existenziellen Analyse und Logotherapie abgeschlossen. Da EMDR für den Einsatz in der Klinik interessant und von der WHO anerkannt ist, habe ich mich auch darin ausbilden lassen. Mein Werdegang umfasst also verschiedene Hintergründe.

Arbeiten Sie als freie Psychotherapeutin in Ihrer eigenen Praxis und/oder (gوf. zusätzlich) als delegierte Psychotherapeutin?

Ich arbeite freiberuflich als Psychotherapeutin, unabhängig und in Delegation mit einem psychiatrischen Arzt. Diese beiden Arbeitsweisen sind interessant für mich und scheinen sich je nach Situation zu vervollständigen. Manche Patient ${ }^{\star}$ innen benötigen ja Medikamente, und die direkte Verbindung mit einem psychiatrischen Arzt, der diese verschreiben kann, vereinfacht die Dinge.
Haben Sie neben der Psychotherapie noch einen weiteren Beruf, eine weitere Beschäftigung?

Da ich einen Doktor in klinischer Psychologie habe, unterrichte ich Entwicklungs- und Lernpsychologie im Rahmen der Lehrerausbildung. Früher am Institut für Heilpädagogik und seit 2003 an der Pädagogischen Hochschule von Fribourg.

Worauf haben Sie sich spezialisiert?

Ich liebe die Vielseitigkeit der Ansätze und habe mich in viele Richtungen hin weitergebildet mit Hinblick auf einen integrierten Humanismus. Aber ich habe mich ausgiebig mit den Fragen der «Spiritual Care» aus dem angelsächsischen Raum befasst und mich in Logotherapie weitergebildet. Die Sinnsuche ist für meinen Ansatz grundlegend. Meine Arbeit orientiert sich an den Ressourcen der Patient*innen und ruht auf einem psychoanalytischen Fundament mit psychosomatischer und noetischer Ausrichtung.

\section{Sind Sie mit Ihrer beruflichen Situation zufrieden?}

Sehr zufrieden, ja, auch wenn ich manchmal wegen meiner verschiedenen Aufgaben etwas zu viel Arbeit habe. So bleibe ich jedoch flexibel und offen. Es gibt mir Elan und Frische, auch wenn ich bald am Ende meiner Karriere angekommen bin.

\section{Wünschen Sie sich, dass etwas anders}

wäre?

Ich hätte gern, dass unser Beruf mehr Anerkennung erhält. Die anstehende Reform geht in die richtige Richtung, aber ich fürchte einerseits eine bürokratische Überlastung durch die ganzen Berichte, die verlangt werden, und andererseits sinkende Honorare. Ich würde mir wünschen, dass die Leistung der Psychotherapie vollkommen anerkannt und ihrer Bedeutsamkeit entsprechend entlohnt wird.

\section{Möchten Sie der ASP etwas mitteilen?}

Ich wünsche mir, dass die ASP mehr Gewicht erhält und dass sie zu einem wirklich einflussreichen Ansprechpartner an der Seite der FSP wird, die in der Romandie stark vertreten ist. Ich weiss den menschlichen Umgang und den Servicegedanken zu schätzen, den ich in der 
ASP wahrnehme. Es ist mir wichtig, dass unsere Dachorganisation bestehen bleibt und sich sogar weiterentwickelt angesichts des besonderen Platzes, den sie einnimmt. Da sie für diverse Bildungshintergründe offen ist, vereint die Assoziation viele Menschen mit unterschiedlichen Lebensläufen aus ganz unterschiedlichen Bereichen, was ein grosser Vorteil ist.

\section{Fühlen Sie sich in Ihrem Berufsverband, der ASP, vertreten und anerkannt?}

Ja, auch wenn sie nicht so viel Gewicht hat wie andere Dachorganisationen, gerade in meiner Region. Ich würde mir wünschen, dass die ASP in der Romandie mehr Gewicht hat und den nicht-deutschsprachigen Regionen der Schweiz mehr Bedeutung zuteilt. Die Sitzungen finden oft in Zürich oder noch weiter weg statt, und deswegen ist es für Leute aus der Romandie fast unmöglich, einfach dorthin zu kommen. Zentraler gelegene Orte würden ein deutliches Signal setzen.

\section{Wo läge Ihre Priorität, wenn Sie}

Vorstandsmitglied der ASP wären?

Der ASP Kraft und Gewicht zu verleihen. Und ich würde mich bemühen, wie bereits erwähnt, gut erreichbare Standorte zu finden, damit jedes Mitglied, egal wo es tätig ist, sich als vollwertiges Mitglied der ASP fühlt.

\section{Gibt es eine Funktion in der ASP,}

die Sie gern ausüben möchten?

Da sich meine berufliche Laufbahn ihrem Ende nähert und aufgrund meiner vielen Verpflichtungen, nein.

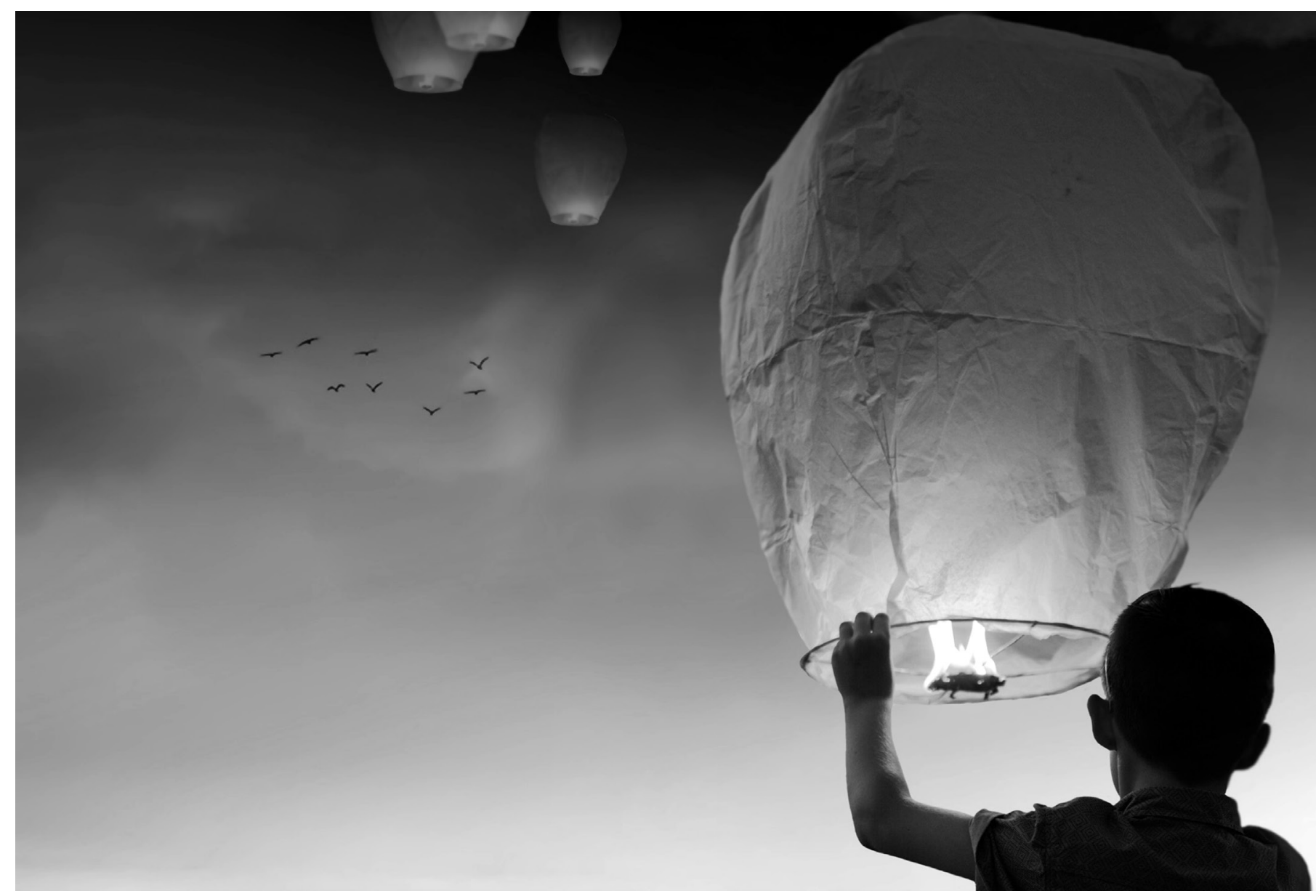


Wie würde Ihrer Meinung nach im gegebenen politischen Umfeld die ideale Situation für Psychotherapeut*innen aussehen?

Eine tatsächliche liberale Anerkennung mit der Möglichkeit, den Beruf gegen eine Honorierung auszuüben, die dem langen Ausbildungsweg der Psychotherapie entspricht.

Welche Vision haben Sie

für Ihren beruflichen Alltag?

Das Ziel besteht darin, vollkommen im Hier und Jetzt der Beziehung zu den Patient*innen sein zu können, das jeweilige Gegenüber zu respektieren, aber auch sich selbst. Und ich freue mich, demnächst beim internationalen Kolloquium der «Clinique du Sens» eine Rede zu halten, das am 14. und 15. November auf dem Universitätscampus in Lausanne stattfindet (https://www. unil.ch/issr/clinique-du-sens).

Elisabeth Ansen Zeder, Dr.in der Psychologie, ist Psychologin der FSP und Psychotherapeutin der ASP, Psychotherapeutin, Ausbilderin und Forscherin sowie seit 2013 Mitglied der ASP. Sie betreibt eine medizinische Psychotherapiepraxis. URLs: www.relationaide.ch | https://www. hepfr.ch/users/elisabethzederfreducanet2ch

Das Gespräch wurde von Sandra Feroleto geführt und schriftlich festgehalten. 